\title{
Enantioselective total synthesis of (+)-panepophenanthrin, a novel inhibitor of the ubiquitin-activating enzyme
}

\author{
Goverdhan Mehta* and Senaiar S. Ramesh \\ Department of Organic Chemistry, Indian Institute of Science, Bangalore 560 012, India
}

\begin{abstract}
An enantioselective total synthesis of the novel natural product (+)-panepophenanthrin has been accomplished in which a biomimetic Diels-Alder dimerisation is a key step. The monomeric precursor $\mathbf{2}$ was assembled from the readily available DielsAlder adduct of cyclopentadiene and $p$-benzoquinone through a short, simple sequence employing chemo- and stereoselective operations.
\end{abstract}

In 2002, Sekizawa et al. reported the isolation of a novel natural product panepophenanthrin from the fermentation broth of the mushroom strain Panus rudis Fr. IFO8994 and assigned it the stereostructure $\mathbf{1}$ on the basis of complementary spectral data and X-ray crystal structure determination. ${ }^{1}$ Concurrently, these authors also observed that panepophenanthrin 1 was the first known inhibitor of the ubiquitin-activating enzyme (E1), which has the pivotal role in activating the ubiquitin-proteasome pathway (UPP). ${ }^{1}$ Since, UPP is involved in the regulation of a number of cellular functions through the degradation/processing of target proteins, the discovery of an inhibitor of the key enzyme (E1) involved in this pathway was of great biological promise in the context of diseases like cancer, inflammation and neurodegenerative disorders. ${ }^{2}$

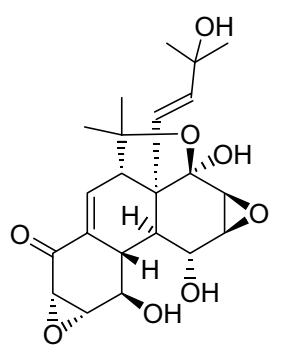

$(+)-1$

\footnotetext{
* Corresponding author. Tel.: +91-80-3602367; fax: +91-80-3600936; e-mail: gm@orgchem.iisc.ernet.in
}

Panepophenanthrin 1 belongs to the general and growing class of epoxyquinoid natural products that are produced through a biosynthetic Diels-Alder reaction. ${ }^{3}$ Other recent examples of dimeric epoxyquinoid natural products are epoxyquinol $\mathrm{A}^{3 \mathrm{~b}}$ and $\mathrm{B}^{3 \mathrm{c}}$ and torreyanic $\mathrm{acid}^{3 \mathrm{~d}}$ and have already aroused considerable synthetic interest. ${ }^{4}$

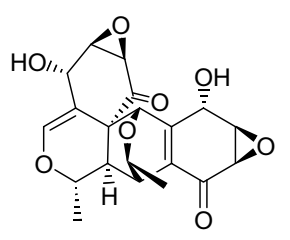

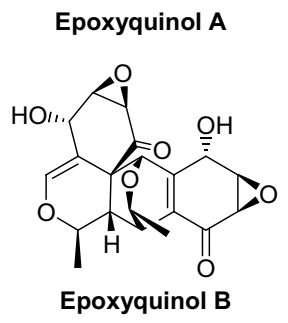

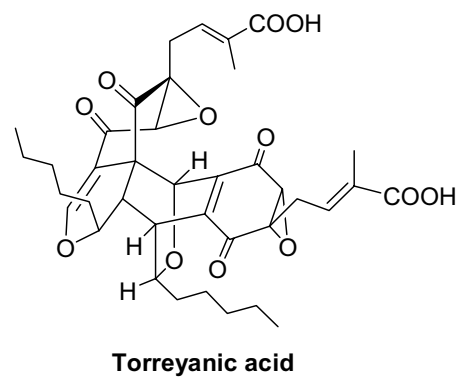

Panepophenanthrin possesses a unique tetracyclic architecture embellished with dense oxygen functionalisation and embodies 11 contiguous stereogenic centres. These structural attributes combined with its interesting biosynthetic origin and biological potential, makes 1 an attractive and formidable synthetic target that has already drawn many synthetic chemists into the fray. Two total syntheses of $\mathbf{1}$, one chiral ${ }^{5}$ and the other 
racemic, ${ }^{6}$ have appeared in the past few months and herein we report a new enantioselective synthesis of $(+)$ 1. Our synthetic approach to $\mathbf{1}$ was inspired by its possible biosynthetic origin, which could involve the stereoselective dimerisation of monomeric epoxyquinoid 2 through a Diels-Alder reaction (3, Scheme 1). This premise drew credence from the recent report of the isolation of panepoxydon $\mathbf{4}$, which on mild acid catalysed allylic rearrangement gave $\mathbf{2}^{7}$ Thus, we identified $\mathbf{2}$ as the pre-target and sought to access it from the advanced precursor (-)-5, Scheme 1. It may be noted that the Diels-Alder based dimerisation strategy adopted here closely follows that described recently by Porco and co-workers. ${ }^{5}$

Our synthesis of $\mathbf{1}$, like that of the related epoxyquinoid derived dimeric natural products epoxyquinols $\mathrm{A}$ and $\mathrm{B},{ }^{4 \mathrm{a}}$ emanated from the readily available tricyclic DielsAlder adduct $6^{8}$ of cyclopentadiene and $p$-benzoquinone, which was further transformed to the endo, endo, meso-diol 7. Lipase mediated enzymatic desymmetrisation of 7 as described previously led to acetate $(+)-8,{ }^{9}$ Scheme 2. Routine functional group transformations in 8 furnished the epoxyketone (+)-10 through stereoselective epoxidation from the exo-face in the enone (+)9, Scheme 2. $\alpha, \beta$-Epoxyketone $\mathbf{1 0}$ on base mediated hydroxymethylation led to (-)-11 and established the

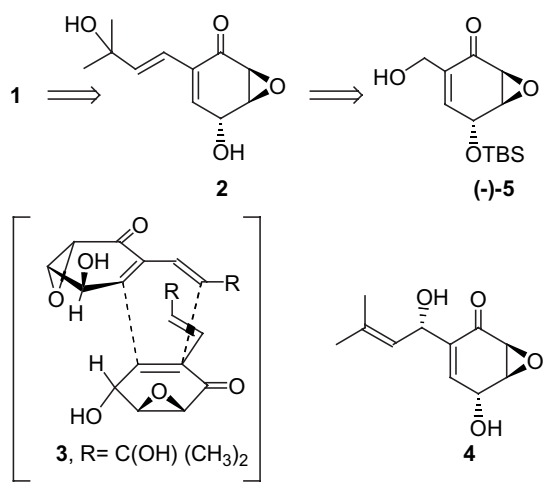

Scheme 1. Retrosynthetic analysis of $\mathbf{1}$.

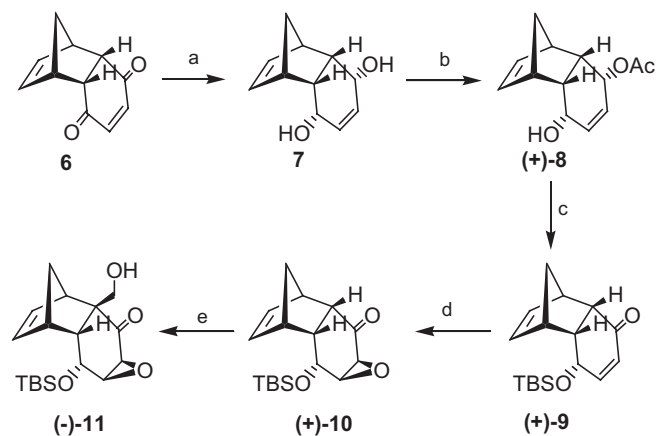

Scheme 2. Reagents and conditions: (a) $\mathrm{NaBH}_{4}, \mathrm{CeCl}_{3}, 0{ }^{\circ} \mathrm{C}, 92 \%$; (b) lipasePS (amano), vinyl acetate, THF, rt, 89\%; (c) (i) TBSCl, imidazole, DMAP, $\mathrm{CH}_{2} \mathrm{Cl}_{2}, \mathrm{rt}, 85 \%$, (ii) $\mathrm{K}_{2} \mathrm{CO}_{3}, \mathrm{MeOH}$, rt, (iii) $\mathrm{PDC}$, $\mathrm{CH}_{2} \mathrm{Cl}_{2}, \mathrm{rt}, 87 \%$ (two steps); (d) $30 \% \mathrm{H}_{2} \mathrm{O}_{2}, \mathrm{Na}_{2} \mathrm{CO}_{3}$, acetone, rt, $67 \%$; (e) DBU, THF, $0{ }^{\circ} \mathrm{C}$ then $35 \%$ formalin, rt, $85 \%$.

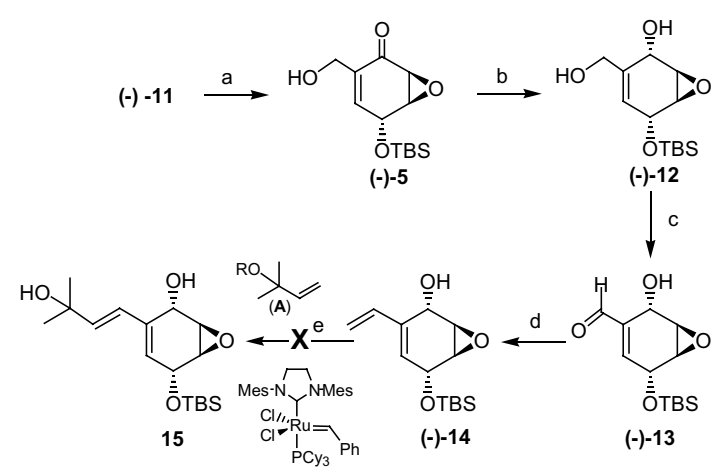

Scheme 3. Reagents and conditions: (a) $\mathrm{Ph}_{2} \mathrm{O}, 240{ }^{\circ} \mathrm{C}, 85 \%$; (b) DIBAL-H ( $1 \mathrm{M}$ in hexane), THF, $-78^{\circ} \mathrm{C}, 10 \mathrm{~min}, 80 \%$; (c) $\mathrm{O}_{2}$, TEMPO, $\mathrm{CuCl}, \mathrm{DMF}, \mathrm{rt}, 96 \%$; (d) $\mathrm{Ph}_{3} \mathrm{P}^{+} \mathrm{CH}_{3} \mathrm{Br}^{-}, \mathrm{KO}^{t} \mathrm{Bu}, \mathrm{THF}, 0{ }^{\circ} \mathrm{C}, 75 \%$; (e) A $(\mathrm{R}=\mathrm{H}$, TBS $)$, second generation Grubbs' catalyst $(5-10 \mathrm{~mol} \%)$, $\mathrm{CH}_{2} \mathrm{Cl}_{2}, 45^{\circ} \mathrm{C}$ or sealed tube, $\mathrm{CH}_{2} \mathrm{Cl}_{2}, 100^{\circ} \mathrm{C}$.

key $\mathrm{C}-\mathrm{C}$ bond required for the elaboration of the side arm in 2. Retro Diels-Alder reaction in $\mathbf{1 1}$ cleanly removed the elements of cyclopentadiene and furnished the enantiomer $(-)-\mathbf{5}$, Scheme $3 .^{9 b}$ DIBAL-H reduction of the carbonyl group in $(-)-5$ proceeded under chelation control ${ }^{10}$ with epoxy oxygen participation and led to the diol (-)-12 ${ }^{11}$ as a single diastereomer. The primary hydroxyl group in $\mathbf{1 2}$ was neatly and chemoselectively oxidised to (-) $\mathbf{1 3}^{11}$ with the TEMPO- $\mathrm{O}_{2}-\mathrm{CuCl}^{12}$ milieu and further Wittig olefination furnished $(-)-\mathbf{1 4},{ }^{11}$ Scheme 3. At this stage, we sought to carry out cross metathesis (CM) between $\mathbf{1 4}$ and 2-methyl-3-buten-2-ol, employing the second generation Grubbs' catalyst $1^{13}$ to furnish $\mathbf{1 5}$, the precursor of $\mathbf{2}$, Scheme 3 . However, despite many efforts and variations this $\mathrm{CM}$ was unsuccessful and necessitated an alternative approach.

Hydroxy-aldehyde (-)-13 was subjected to a HornerWittig olefination to furnish exclusively and in good yield the $(E)-\alpha, \beta$-unsaturated ester $(-)-16,{ }^{11}$ Scheme 4. Addition of an excess of methyl lithium to $\mathbf{1 6}$ led smoothly to the desired tertiary alcohol $(-)-15 .^{11}$ Oxidation of the allylic hydroxyl group with $\mathrm{MnO}_{2}$ furnished dienone (-)-17. ${ }^{11}$ Deprotection of the TBS group in $\mathbf{1 7}$ led to 2, our pre-target and the monomeric precursor for the biomimetic Diels-Alder reaction to $\mathbf{1}$.

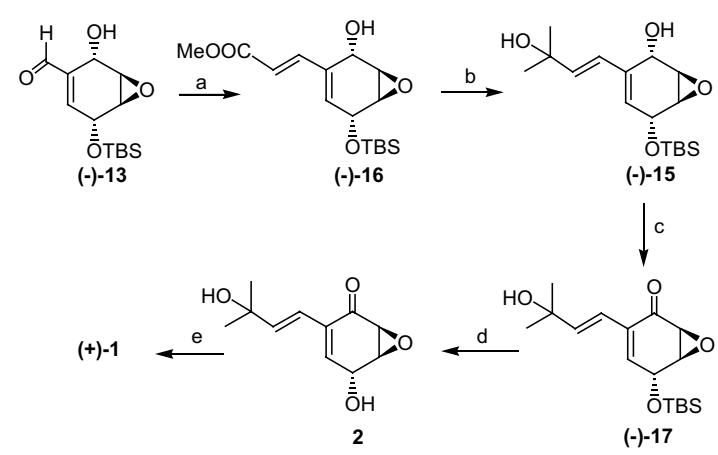

Scheme 4. Reagents and conditions: (a) $\mathrm{Ph}_{3} \mathrm{P}=\mathrm{CHCOOMe}, \mathrm{C}_{6} \mathrm{H}_{6}$, rt, $91 \%$; (b) $\mathrm{MeLi}$, THF, $0{ }^{\circ} \mathrm{C}, 56 \%$; (c) $\mathrm{MnO}_{2}, \mathrm{CH}_{2} \mathrm{Cl}_{2}, \mathrm{rt}, 74 \%$; (d) $\mathrm{HF}-$ pyridine, THF, rt, quant.; (e) neat, rt, $24 \mathrm{~h}, 74 \%$. 
When 2 was left aside without solvent for $24 \mathrm{~h}$, it was neatly transformed into a single polar product through the desired stereospecific intermolecular Diels-Alder reaction (see 3). ${ }^{14}$ The spectral data $\left({ }^{1} \mathrm{H}\right.$ and ${ }^{13} \mathrm{C}$ NMR) and specific rotation $\left([\alpha]_{\mathrm{D}}^{24}=+147.2^{\circ}(\right.$ c $0.91, \mathrm{MeOH})$, lit. $\left.{ }^{1}:[\alpha]_{\mathrm{D}}^{26}=+149.8^{\circ}(c 1.0, \mathrm{MeOH})\right)$ for synthetic 1 were found to be identical with the natural product panepophenanthrin $(+)-1 .{ }^{1}$

In short, we have accomplished an enantioselective total synthesis of panepophenanthrin $\mathbf{1}$, the novel inhibitor of the enzyme E1 of the ubiquitin-proteasome pathway from the readily and abundantly available Diels-Alder adduct of cyclopentadiene and $p$-benzoquinone. Our approach is amenable to enantiodivergency and is flexible to render access to analogues for further biological evaluation.

\section{Acknowledgements}

This research was supported by the Chemical and Biology Unit of JNCASR, Bangalore, India. One of us (S.S.R), would like to thank the CSIR (India) for the award of a research fellowship. We also thank Mr. Kabirul Islam (IISc) for useful discussions and valuable suggestions.

\section{References and notes}

1. Sekizawa, R.; Ikeno, S.; Nakamura, H.; Naganawa, H.; Matsui, S.; Iinuma, H.; Takeuchi, T. J. Nat. Prod. 2002, $65,1491$.

2. Swinney, D. C. Drug Discovery Today 2001, 6, 244.

3. (a) Review: Williams, R. M.; Stocking, E. M. Angew. Chem., Int. Ed. 2003, 42, 3078; (b) Recent examples: Kakeya, H.; Onose, R.; Koshino, H.; Yoshida, A.; Kobayashi, K.; Kageyama, S.-I.; Osada, H. J. Am. Chem. Soc. 2002, 124, 3496; (c) Kakeya, H.; Onose, R.; Yoshida, A.; Koshino, H.; Osada, H. J. Antibiot. 2002, 55, 829; (d) Lee, J. C.; Strobel, G. A.; Lobkovsky, E.; Clardy, J. J. Org. Chem. 1996, 61, 3232.

4. (a) Mehta, G.; Islam, K. Tetrahedron Lett. 2003, 44, 3569; (b) Shoji, M.; Yamaguchi, J.; Kakeya, H.; Osada, H.; Hayashi, Y. Angew. Chem., Int. Ed. 2002, 41, 3192; (c) Li, C.; Bardhan, S.; Pace, E. A.; Liang, M.-C.; Gilmore, T. D.; Porco, J. A., Jr. Org. Lett. 2002, 4, 3267.

5. Lei, X.; Johnson, R. P.; Porco, J. A., Jr. Angew. Chem., Int. Ed. 2003, 42, 3913.

6. Moses, J. E.; Commeiras, L.; Baldwin, J. E.; Adlington, R. M. Org. Lett. 2003, 5, 2987.

7. Shotwell, J. B.; Hu, S.; Medina, E.; Abe, M.; Cole, R.; Crews, M. C.; Wood, J. L. Tetrahedron Lett. 2000, 41, 9639.

8. Cookson, R. C.; Crundwell, E.; Hill, R. R.; Hudec, J. J. Chem. Soc. 1964, 3062.
9. (a) Talano, S.; Higashi, Y.; Kamikubo, T.; Moriya, M.; Ogasawara, K. Synthesis 1993, 948; (b) Kamikubo, T.; Hiroya, K.; Ogasawara, K. Tetrahedron Lett. 1996, 37, 499.

10. Kiyooka, S.; Kuroda, H.; Shimasaki, Y. Tetrahedron Lett. 1986, 27, 3009.

11. All compounds were fully characterised on the basis of IR, ${ }^{1} \mathrm{H}$ NMR, ${ }^{13} \mathrm{C}$ NMR, mass data. Selected spectral data $(-)-12, \quad[\alpha]_{\mathrm{D}}^{23}=-34.5^{\circ} \quad\left(c \quad 0.55, \quad \mathrm{CHCl}_{3}\right) ;{ }^{1} \mathrm{H} \quad \mathrm{NMR}$ $\left(300 \mathrm{MHz}, \mathrm{CDCl}_{3}\right): \delta 5.61(\mathrm{~d}, J=3.3 \mathrm{~Hz}, 1 \mathrm{H}), 4.52(\mathrm{~d}$, $J=3.3 \mathrm{~Hz}, 1 \mathrm{H}), 4.37$ (br s, $1 \mathrm{H}), 4.23 \quad(1 / 2 \mathrm{ABq}$, $J=13.8 \mathrm{~Hz}, 1 \mathrm{H}), 4.14(1 / 2 \mathrm{ABq}, J=13.8 \mathrm{~Hz}, 1 \mathrm{H}), 3.39$ (m, 1H), $3.21(\mathrm{~m}, 1 \mathrm{H}), 2.77$ (br s, OH), 2.45 (br s, OH), $0.92(\mathrm{~s}, 9 \mathrm{H}), 0.16(\mathrm{~s}, 3 \mathrm{H}), 0.15(\mathrm{~s}, 3 \mathrm{H}) ;{ }^{13} \mathrm{C} \mathrm{NMR}(75 \mathrm{MHz}$, $\left.\mathrm{CDCl}_{3}\right): \delta 137.1,121.9,64.9,63.7,63.6,53.1,52.6,25.8$, $18.2,-4.62,-4.79$; HRMS (ES) $\mathrm{m} / z$ calcd for $\mathrm{C}_{13} \mathrm{H}_{24} \mathrm{NaO}_{4} \mathrm{Si}, \quad[\mathrm{M}+\mathrm{Na}]^{+}:$295.1342, found: 295.1347; $(-)-16 \quad[\alpha]_{\mathrm{D}}^{23}=-87.8^{\circ} \quad\left(c \quad 0.41, \quad \mathrm{CHCl}_{3}\right) ;{ }^{1} \mathrm{H} \quad \mathrm{NMR}$ $\left(300 \mathrm{MHz}, \mathrm{CDCl}_{3}\right): \delta 7.23(\mathrm{~d}, J=16.2 \mathrm{~Hz}, 1 \mathrm{H}), 6.23(\mathrm{~d}$, $J=16.2 \mathrm{~Hz}, 1 \mathrm{H}), 5.93(\mathrm{~d}, J=4.5 \mathrm{~Hz}, 1 \mathrm{H}), 4.66(\mathrm{~d}$, $J=10.2 \mathrm{~Hz}, 1 \mathrm{H}), 4.62(\mathrm{~d}, J=4.5 \mathrm{~Hz}, 1 \mathrm{H}), 3.76(\mathrm{~s}, 3 \mathrm{H})$, 3.49 (br s, $1 \mathrm{H}), 3.26$ (br s, $1 \mathrm{H}), 2.28$ (d, $J=10.2 \mathrm{~Hz}, \mathrm{OH})$, $0.91(\mathrm{~s}, 9 \mathrm{H}), 0.16(\mathrm{~s}, 3 \mathrm{H}), 0.14(\mathrm{~s}, 3 \mathrm{H}) ;{ }^{13} \mathrm{C} \mathrm{NMR}(75 \mathrm{MHz}$, $\left.\mathrm{CDCl}_{3}\right): \delta 167.2,144.2,134.1,133.2,119.7,63.8,62.1$, 52.8, 52.0, 51.7, 25.7, 18.2, -4.57, -4.76; HRMS (ES) $\mathrm{m} / \mathrm{z}$ calcd for $\mathrm{C}_{16} \mathrm{H}_{26} \mathrm{NaO}_{5} \mathrm{Si}[\mathrm{M}+\mathrm{Na}]^{+}:$349.1447, found: 349.1435; (-)-15 $[\alpha]_{\mathrm{D}}^{23}=-84.8^{\circ} \quad\left(c \quad 0.66, \mathrm{CHCl}_{3}\right) ;{ }^{1} \mathrm{H}$ NMR $\left(300 \mathrm{MHz}, \mathrm{CDCl}_{3}\right): \delta 6.18(\mathrm{~d}, J=16.2 \mathrm{~Hz}, 1 \mathrm{H})$, $6.12(\mathrm{~d}, J=16.2 \mathrm{~Hz}, 1 \mathrm{H}), 5.60(\mathrm{dd}, J=5.4,1.8 \mathrm{~Hz}, 1 \mathrm{H})$, 4.67 (br s, 1H), 4.58 (dd, $J=5.4,1.8 \mathrm{~Hz}, 1 \mathrm{H}), 3.48(\mathrm{~m}$, $1 \mathrm{H}), 3.24(\mathrm{~m}, 1 \mathrm{H}), 1.36(\mathrm{~s}, 3 \mathrm{H}), 1.35(\mathrm{~s}, 3 \mathrm{H}), 0.92(\mathrm{~s}, 9 \mathrm{H})$, $0.16(\mathrm{~s}, 3 \mathrm{H}), 0.14(\mathrm{~s}, 3 \mathrm{H}) ;{ }^{13} \mathrm{C}$ NMR $\left(75 \mathrm{MHz}, \mathrm{CDCl}_{3}\right): \delta$ 138.8, 135.3, 126.2, 125.8, 70.9, 64.1, 62.5, 52.9, 52.1, 29.8, 29.7, 25.8, 18.2, -4.5, -4.7; HRMS (ES) $\mathrm{m} / \mathrm{z}$ calcd for $\mathrm{C}_{17} \mathrm{H}_{30} \mathrm{NaO}_{4} \mathrm{Si}[\mathrm{M}+\mathrm{Na}]^{+}: 349.1811$, found 349.1818; (-)17, $[\alpha]_{\mathrm{D}}^{23}=-184.8^{\circ}\left(c 0.92, \mathrm{CHCl}_{3}\right) ;{ }^{1} \mathrm{H}$ NMR $(400 \mathrm{MHz}$, $\left.\mathrm{CDCl}_{3}\right): \delta 6.43(\mathrm{~m}, 2 \mathrm{H}), 6.3(\mathrm{~d}, J=16.2 \mathrm{~Hz}, 1 \mathrm{H}), 4.74(\mathrm{~d}$, $J=3.9 \mathrm{~Hz}, 1 \mathrm{H}), 3.65(\mathrm{~m}, 1 \mathrm{H}), 3.54(\mathrm{dd}, J=3.7,1 \mathrm{~Hz}$, $1 \mathrm{H}), 1.37(\mathrm{~s}, 3 \mathrm{H}), 1.35(\mathrm{~s}, 3 \mathrm{H}), 0.92(\mathrm{~s}, 9 \mathrm{H}), 0.18(\mathrm{~s}, 3 \mathrm{H})$, 0.15 (s, 3H). ${ }^{13} \mathrm{C}$ NMR (100 MHz, $\left.\mathrm{CDCl}_{3}\right): \delta 193.4,142.4$, 138.2, 132.9, 119.9, 71.1, 64.2, 58.1, 54.3, 29.7 (2C), 25.7, $18.2,-4.3,-4.5$; HRMS (ES) $m / z$ calcd for $\mathrm{C}_{17} \mathrm{H}_{28} \mathrm{NaO}_{4} \mathrm{Si}$ $[\mathrm{M}+\mathrm{Na}]^{+}: \quad 347.1655$, found: 347.1676; (+)-1, $[\alpha]_{\mathrm{D}}^{24}=+147.2^{\circ}($ c $0.91, \mathrm{MeOH}) ;{ }^{1} \mathrm{H} \mathrm{NMR}(400 \mathrm{MHz}$, $\left.\mathrm{CDCl}_{3}\right): \delta 6.81(\mathrm{dd}, J=5.0,3.0 \mathrm{~Hz}, 1 \mathrm{H}), 5.99 \quad(\mathrm{~d}$, $J=16.2 \mathrm{~Hz}, 1 \mathrm{H}), 5.68(\mathrm{~d}, J=16.2 \mathrm{~Hz}, 1 \mathrm{H}), 4.55$ (br s, $1 \mathrm{H}), 4.35(\mathrm{br} \mathrm{s}, 1 \mathrm{H}), 3.84(\mathrm{t}, J=3.4 \mathrm{~Hz}, 1 \mathrm{H}), 3.50(\mathrm{t}$, $J=3.2 \mathrm{~Hz}, 1 \mathrm{H}), 3.42(\mathrm{~d}, J=4.0 \mathrm{~Hz}, 1 \mathrm{H}), 3.35(\mathrm{dd}$, $J=5.0,1.6 \mathrm{~Hz}, 1 \mathrm{H}), 3.31(\mathrm{~d}, J=4 \mathrm{~Hz}, 1 \mathrm{H}), 2.32(\mathrm{br} \mathrm{d}$, $J=10.0 \mathrm{~Hz}, 1 \mathrm{H}), 2.03($ br d, $J=9.7 \mathrm{~Hz}, 1 \mathrm{H}), 1.45(\mathrm{~s}, 3 \mathrm{H})$, $1.35(\mathrm{~s}, 3 \mathrm{H}), 1.20(\mathrm{~s}, 3 \mathrm{H}), 1.17(\mathrm{~s}, 3 \mathrm{H}) .{ }^{13} \mathrm{C} \mathrm{NMR}(75 \mathrm{MHz}$, $\left.\mathrm{CDCl}_{3}\right): \delta 196.3,143.0,139.9,138.8,129.3,102.7,79.2$, 71.8, 69.0, 66.2, 60.7, 57.4, 57.2, 57.1, 55.6, 55.1, 51.2, 50.0, $32.3,30.3,29.5,26.2$; HRMS (ES) $\mathrm{m} / z$ calcd for $\mathrm{C}_{22} \mathrm{H}_{28} \mathrm{NaO}_{8}[\mathrm{M}+\mathrm{Na}]^{+}$: 443.1682, found: 443.1698.

12. Semmelhack, M. F.; Schmid, C. R.; Cortes, D. A.; Chou, C. S. J. Am. Chem. Soc. 1984, 106, 3374.

13. Chatterjee, A. K.; Grubbs, R. H. Org. Lett. 1999, 1, 1751.

14. The stereoselectivity in the Diels-Alder dimerisation of $\mathbf{2}$ leading to the natural product $\mathbf{1}$ is quite remarkable and several explanations have been offered ${ }^{5,6}$ and elegantly probed $^{5}$ both experimentally and computationally, particularly by the group of Porco. 